10

\title{
Особенности спектрально-разрешенной термолюминесценции в облученных микрокристаллах нитрида алюминия
}

\author{
() Д.М. Спиридонов ${ }^{1}$, Д.В. Чайкин ${ }^{1}$, Н.А. Мартемьянов ${ }^{1}$, A.C. Вохминцев $^{1}$, И.А. Вайнштейн ${ }^{1,29}$ \\ ${ }^{1}$ НОЦ НАНОТЕХ, Уральский федеральный университет, \\ 620002 Екатеринбург, Россия \\ ${ }^{2}$ Институт металлургии Уральского отделения РАН, \\ 620016 Екатеринбург, Россия \\ I e-mail: i.a.weinstein@urfu.ru
}

Поступила в редакцию 11.02.2020 г.

В окончательной редакции 11.02.2020 г.

Принята к публикации 28.03.2020 г.

Исследованы закономерности процессов фото- и термолюминесценции в субмикронных кристаллах AlN с катионным дефицитом после возбуждения УФ излучением. Наблюдаемые спектры свечения представляют собой суперпозицию полос с максимумами при 3.0 и $2.5 \mathrm{eV}$. Указанные спектральные особенности обусловлены электронными переходами с участием примесных $\mathrm{O}_{\mathrm{N}}$ и кислород-вакансионных центров типа $\left(V_{\mathrm{Al}}-\mathrm{O}_{\mathrm{N}}\right)$. Центры захвата носителей заряда на основе азотных вакансий $V_{\mathrm{N}}$, согласно количественному анализу в рамках описания кинетических процессов общего порядка, имеют энергию активации $0.45 \mathrm{eV}$ и отвечают за формирование термоактивационного пика при температуре $345 \mathrm{~K}$.

Ключевые слова: AlN, фотолюминесценция, термолюминесценция, энергия активации, кислород-связанный центр, вакансия.

DOI: $10.21883 /$ OS.2020.09.49872.43-20

\section{Введение}

В настоящее время большое внимание уделяется созданию микро- и наноструктур на основе III-нитридов с различными морфологическими особенностями, а также исследованию их свойств применительно к решению разнообразных прикладных задач опто- и наноэлектроники. Немаловажное место среди подобных материалов занимает нитрид алюминия благодаря своей широкой запрещенной зоне около $6.2 \mathrm{eV}$, химической стойкости, высокой теплопроводности и т.д. [1-7]. Известны применения AlN для создания светодиодов и устройств поверхностных акустических волн, подложек для элементов электроники, детекторных сред для регистрации ионизирующего излучения различной природы [8-14]. Кроме того, микро- и наноструктуры AlN используются для создания компактных устройств на базе микролазеров УФ и видимого диапазонов [8,15-18].

Технологические методы и способы получения AlN имеют серьезные ограничения. Большая часть из них (выращивание с хлоридом, реакция с углеродными нанотрубками, дуговое разряжение, каталитический рост с использованием диоксида кремния и рост паржидкость-твердое тело) характеризуются низкой производительностью, высокой стоимостью прекурсоров и большим содержанием примесей в конечных продуктах [19-25]. В связи с этим поиск новых путей синтеза AlN с возможностью формирования кристаллических структур с заданной геометрией и нужными спектральными характеристикам является актуальной задачей современного оптического материаловедения.
В настоящей работе представлены исследования люминесцентных свойств субмикронных катион-дефицитных кристаллов AlN в виде гексагональных призм, синтезированных по оригинальной методике.

\section{Образцы и методика эксперимента}

В работе исследовались свойства микрокристаллического порошка $\mathrm{AlN}$, синтезированного на оригинальной установке методом газофазного синтеза при одновременной обработке жидкого алюминия газообразными $\mathrm{AlF}_{3}$ и $\mathrm{NH}_{3}$ [26]. На снимке СЭМ (рис. 1) видно, что частицы синтезированного порошка представляют

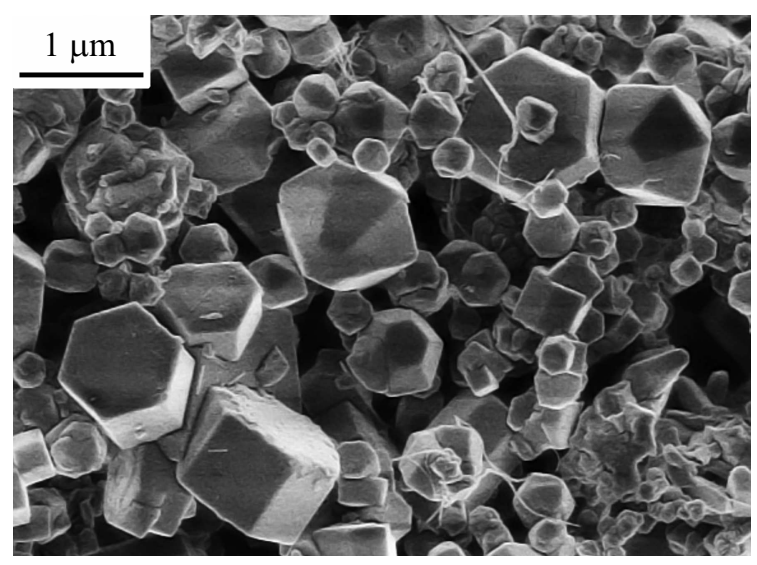

Рис. 1. СЭМ-изображение частиц синтезированного порошка AlN. 
собой гексагональные призмы и комбинации призмы с дипирамидой с характерными размерами $0.1-2.0 \mu \mathrm{m}$. По результатам рентгенофазового анализа [27] установлено наличие единственной кристаллической фазы AlN с пространственной группой вюрцита $P 6_{3} m c$ и параметрами решетки $a=3.1117 \AA$ и $c=4.9794 \AA$. Структура исследуемых частиц характеризуется нестехиометрией по $\mathrm{Al}$ с соотношением $\mathrm{Al}: \mathrm{N}=0.9: 1$, основными примесями являются атомы O (1.6 at.\%) и $\mathrm{Si}(0.5$ at. \%).

Исследование процессов фото- (ФЛ) и термостимулированной люминесценции (ТЛ) проводилось с использованием оригинальной автоматизированной установки на базе спектрометра LS55 Perkin Elmer и высокотемпературной приставки. Принцип работы установки и методика измерений трехмерных ТЛ-зависимостей подробно описаны в работах $[28,29]$. Образцы предварительно отжигались до $650 \mathrm{~K}$.

Спектр ФЛ регистрировался при 1 импульсе возбуждения, задержка до начала измерения составляла $0.05 \mathrm{~ms}$, сбор данных $-12.5 \mathrm{~ms}$ и весь цикл измерения $-20 \mathrm{~ms}$. Возбуждение ФЛ осуществлялось в полосе $265 \mathrm{~nm}$ согласно данным из $[27,30]$. Регистрация сигнала проводилась при сканировании в диапазоне $250-700 \mathrm{~nm}$ со скоростью $120 \mathrm{~nm} / \mathrm{min}$. Ширина щели входного тракта составляла $10 \mathrm{~nm}$, выходного $-20 \mathrm{~nm}$. Для выполнения численной обработки полученные экспериментальные зависимости для интенсивности свечения $I(E)$ были пересчитаны в энергоспектры $I(E)$ по известной методике (см., например, [31]).

Для измерения ТЛ-кривых проводилось предварительное облучение порошка AIN в области $260 \mathrm{~nm}$ в течение $3 \mathrm{~min}$. После этого осуществлялся нагрев от комнатной температуры до $650 \mathrm{~K}$ со скоростью $r=2 \mathrm{~K} / \mathrm{s}$ и проводилась регистрация ТЛ-сигнала в диапазоне $250-650 \mathrm{~nm}$ с шагом $10 \mathrm{~nm}$.

\section{Результаты}

На рис. 2 видно, что спектр свечения ФЛ в микрокристаллах AlN представляет собой широкую неструктурированную полосу. Максимум регистрируется в области $420 \mathrm{~nm}$. В диапазоне $470-490 \mathrm{~nm}$ наблюдается небольшое плечо. В спектрах катодо- и термостимулированной люминесценции $[27,30]$ также наблюдается указанная спектральная особенность.

При исследовании процессов термостимулированного свечения в УФ облученных микрокристаллах AlN была построена экспериментальная трехмерная ТЛзависимость в координатах „интенсивность $I$-длина волны-температура $T^{\text {“ }}$ (рис. 3). Видно, что уже при комнатной температуре в образце наблюдается интенсивное послесвечение. При этом сама 3D-зависимость представляет собой одиночный неструктурированный пик с максимумом в полосе $410 \pm 5 \mathrm{~nm}$ при температуре $343 \pm 5 \mathrm{~K}$. Оценка спектральных и кинетических

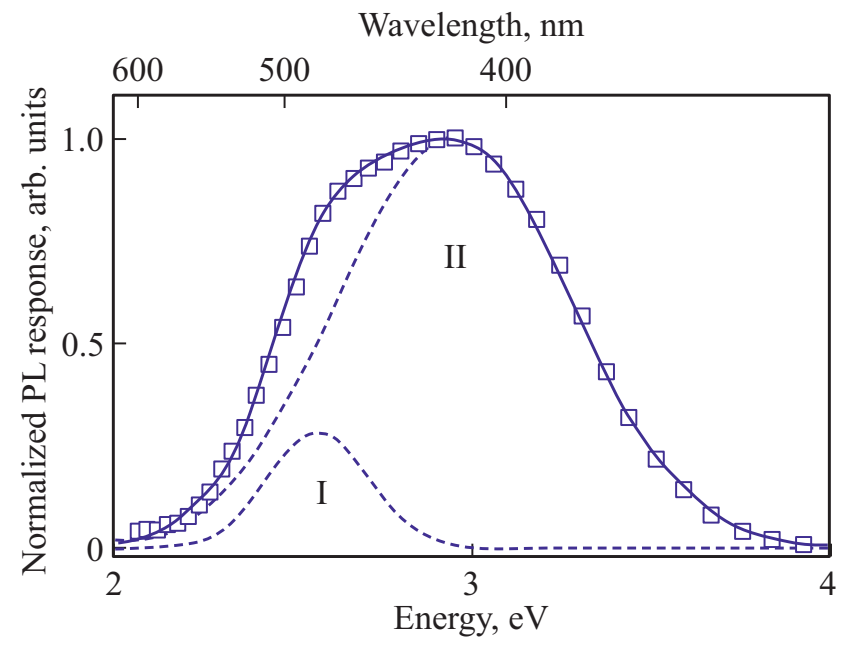

Рис. 2. Спектр ФЛ при возбуждении в полосе $265 \mathrm{~nm}$, результат его аппроксимации (сплошная линия) суммой двух гауссовых кривых I и II (штриховые линии).

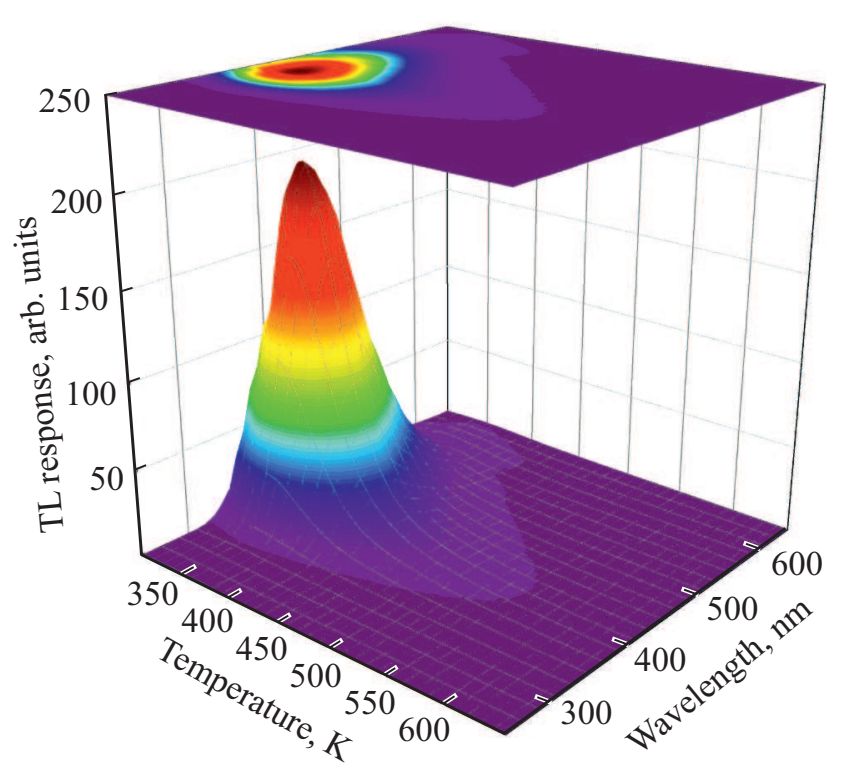

Рис. 3. Спектрально-температурные зависимости ТЛсвечения.

параметров полученных зависимостей и их обсуждение приведены ниже.

\section{Обсуждение}

Измеренные ФЛ- и ТЛ-спектры с высокой степенью точности $\left(R^{2}>0.999\right)$ были аппроксимированы с использованием суперпозиции 2-х независимых гауссовых компонент (рис. 2 и 4, штриховые линии; табл. 1). Видно, что вне зависимости от вида возбуждения, фотоили термоположение полученных полос остается практически неизменным. Доминирующим является широкий пик с максимумом $3.00 \pm 0.05 \mathrm{eV}$. Второй компонентой 
Таблица 1. Спектральные параметры

\begin{tabular}{|c|c|c|c|c|c|}
\hline \multicolumn{2}{|c|}{ Параметры, eV } & \multirow{2}{*}{ ФЛ } & \multirow{2}{*}{ ТЛ } & \multicolumn{2}{|c|}{ Литература } \\
\hline & & & & Полоса, eV & Дефект \\
\hline \multirow{2}{*}{ I } & $E_{\max }$ & $2.57 \pm 0.06$ & $2.43 \pm 0.06$ & \multirow{2}{*}{$2.46-2.54$} & \multirow{2}{*}{$\begin{array}{c}\mathrm{SD} \rightarrow \mathrm{V}_{\mathrm{Al}}-2 \mathrm{O}_{\mathrm{N}}[39] \\
\mathrm{V}_{\mathrm{Al}}-2 \mathrm{O}_{\mathrm{N}}[27,30]\end{array}$} \\
\hline & $\omega_{E}$ & $0.33 \pm 0.05$ & $0.32 \pm 0.05$ & & \\
\hline \multirow[t]{2}{*}{ II } & $E_{\max }$ & $2.95 \pm 0.01$ & $3.04 \pm 0.01$ & \multirow[t]{2}{*}{$2.87-3.1$} & \multirow{2}{*}{$\begin{array}{c}\mathrm{V}_{\mathrm{N}} \rightarrow \mathrm{V}_{\mathrm{Al}}^{3-}-3 \mathrm{O}_{\mathrm{N}}^{+}[37] \\
\mathrm{O}_{\mathrm{N}} \rightarrow\left(\mathrm{V}_{\mathrm{Al}}-\mathrm{O}_{\mathrm{N}}\right)[38] \\
\mathrm{O}_{\mathrm{N}}-\text { комплекс }[32] \\
\mathrm{V}_{\mathrm{Al}}[34] \\
\mathrm{V}_{\mathrm{Al}}^{3-}-\mathrm{O}_{\mathrm{N}}[35,36] \\
\mathrm{O}-\mathrm{DX} \rightarrow \mathrm{V}_{\mathrm{Al}}^{3-}[22,36] \\
\left(\mathrm{V}_{\mathrm{Al}}-\mathrm{O}_{\mathrm{N}}\right)[27,30]\end{array}$} \\
\hline & $\omega_{E}$ & $0.77 \pm 0.01$ & $0.71 \pm 0.01$ & & \\
\hline
\end{tabular}

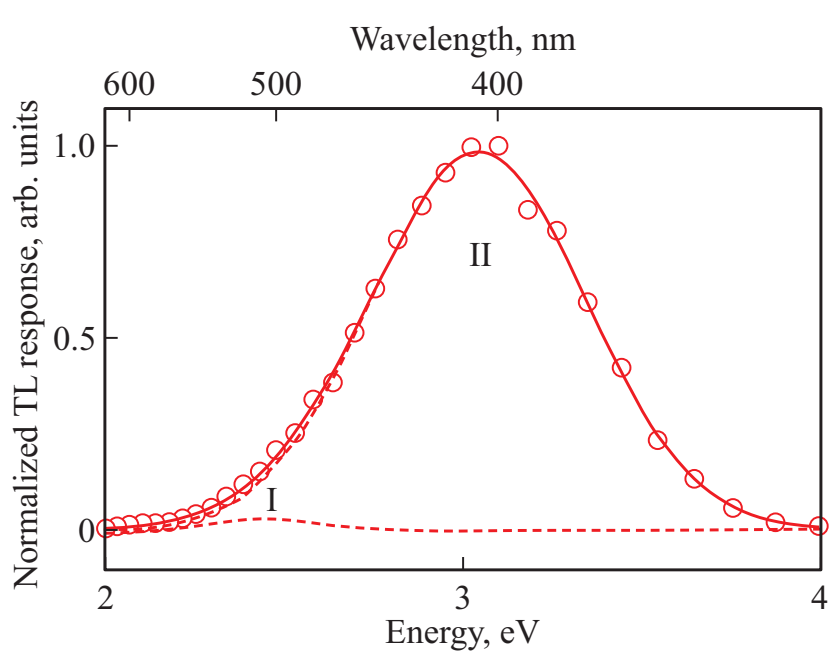

Pис. 4. Спектр ТЛ (штриховая линия - гауссовы компоненты I и II; сплошная линия - результат аппроксимации).

является полоса в области $2.50 \pm 0.05 \mathrm{eV}$. При этом отношение интенсивностей свечения указанных пиков зависит от вида люминесценции. В случае с ТЛ максимальные интенсивности полос различаются в 33 раза, при фотовозбуждении - в 4 раза.

Возможная природа обнаруженных спектральных пиков была проанализирована на основе данных предыдущих наших работ и результатов независимых исследований различных структурных модификаций AlN. Известно [32], что в термостимулированном свечении порошков AlN : O наблюдается ТЛ-пик $2.96 \mathrm{eV}$.

Согласно [23,33,34], КЛ- и ФЛ- максимумы $3 \mathrm{eV}$ в допированных слоях AlN могут быть связаны с наличием излучательных центров на основе вакансий алюминия $V_{\mathrm{Al}}$. Авторы показали, что при увеличении в образцах концентрации примеси $\mathrm{Si}$ интенсивность свечения в данной области возрастала за счет формирования дополнительных $V_{\mathrm{Al}}$-центров. В работах $[35,36]$ КЛ-полоса $3.1 \mathrm{eV}$ в эпитаксиальных слоях AlN была приписана комплексным дефектам типа $V_{\mathrm{Al}}^{3-}-\mathrm{O}$. Кроме того, в $[22,36]$ сделано предположение, что обсуждаемая люминесценция возникает за счет рекомбинационных переходов между O-DX-центрами и комплексами с участием $V_{\mathrm{Al}}^{3-}$. Полоса с максимумом $2.95 \mathrm{eV}$ регистрировалась также при исследованиях ФЛ в нанокристаллических порошках AlN [37] и была отнесена к электроноптическим переходам между уровнями вакансий азота $V_{\mathrm{N}}$ и комплексных дефектов типа $V_{\mathrm{Al}}^{3-}-3 \mathrm{O}_{\mathrm{N}}^{+}$. Ранее нами [38] в объемных монокристаллах AlN также было обнаружено ТЛ-свечение $2.91 \mathrm{eV}$, которое обусловлено рекомбинацией донорно-акцепторных пар на основе нейтральных кислородных примесей в позиции азота $\mathrm{O}_{\mathrm{N}}$ и вакансий алюминия $\left(V_{\mathrm{Al}}-\mathrm{O}_{\mathrm{N}}\right)$.

Вторая спектральная компонента хорошо согласуется со свечением $2.5 \mathrm{eV}(480 \mathrm{~nm})$ в структурах с развитой поверхностью и поликристаллических порошках [39]. В ряде случаев его относят к переходам между уровнями мелких доноров и комплексов вида $V_{\mathrm{Al}}-2 \mathrm{O}_{\mathrm{N}}$. В табл. 1 приведена обобщенная информация о возможной природе оптически активных центров и переходов, ответственных за наблюдаемые спектральные пики.

На рис. 5 даны примеры полученных ТЛ-кривых (спектральных сечений 3D-зависимости). Видно, что их форма $(\mu=0.50-0.52)$ и положение максимума $\left(T_{\max }=342-345 \mathrm{~K}\right)$ практически не меняются в рассматриваемом спектральном диапазоне. Для количественного описания полученных данных использовали известный формализм кинетических процессов общего порядка (GOK) [40]:

$$
I(T)=s^{\prime \prime} n_{0} e^{-\frac{E_{A}}{k T}}\left(1+\frac{s^{\prime \prime}(b-1)}{r} \int_{T_{0}}^{T} e^{-\frac{E_{A}}{k \theta}} d \theta\right)^{-\frac{b}{b-1}},
$$

$s^{\prime \prime}$ - эффективный частотный фактор, $\mathrm{s}^{-1} ; n_{0}$ - начальная концентрация захваченных носителей заряда, $\mathrm{m}^{-3}$; $E_{A}$ - энергия активации, $\mathrm{eV} ; k-$ постоянная Больцмана, $\mathrm{eV} / \mathrm{K} ; b-$ порядок кинетики. 


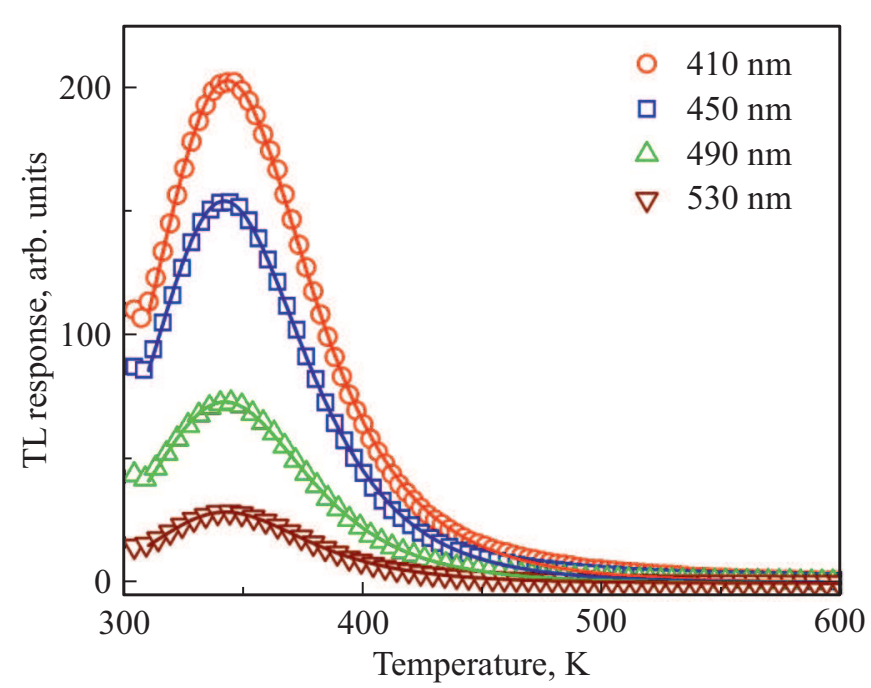

Рис. 5. Кривые ТЛ при регистрации в разных спектральных диапазонах.

Таблица 2. Рассчитанные значения кинетических параметров

\begin{tabular}{c|c}
\hline Параметры & Значения \\
\hline$T_{\max }, \mathrm{K}$ & $341-346$ \\
$\omega_{T}, \mathrm{~K}$ & $74-79$ \\
$\mu$ & $0.50-0.52$ \\
$E_{A}, \mathrm{eV}$ & $0.44-0.46$ \\
$s, \mathrm{~s}^{-1}$ & $(1.25-2.29) \cdot 10^{5}$ \\
$b$ & $2.10-2.31$
\end{tabular}

Результаты аппроксимации представлены на рис. 5 сплошными линиями. Видно, что все кривые ТЛ с высокой степенью точности $\left(R^{2}>0.999\right)$ могут быть описаны одной кинетической компонентой. Полученные параметры кинетики приведены в табл. 2. Оценка энергии активации $E_{A}$ для изучаемого термостимулированного процесса вполне согласуется с результатами для микро- [30] и объемных монокристаллов [38]. В указанных работах параметр $E_{A}=0.45 \pm 0.01 \mathrm{eV}$ характеризует термическую глубину ловушки на основе вакансии азота $V_{\mathrm{N}}$. Полученные значения для порядка кинетики $b$ позволяют сделать вывод о высокой вероятности повторного захвата освободившихся носителей заряда на энергетические уровни в запрещенной зоне, которые активно заполняются и опустошаются в температурном диапазоне исследуемого пика ТЛ.

Можно заключить, что в исследованном микрокристаллическом порошке AlN процессы термостимулированной рекомбинации протекают с участием дефектных комплексов кислород-вакансионной природы. Дефектные уровни вакансий $\left(V_{\mathrm{N}}\right)$ в азотной подрешетке формируют центры захвата электронов и в случае нагрева образца выступают в роли глубокого донора. В свою очередь $\left(V_{\mathrm{Al}}-\mathrm{O}_{\mathrm{N}}\right)$-центры характеризуются сложной структурой основных и возбужденных состояний. Вакансия алю- миния обеспечивает уровни дырочного захвата вблизи валентной зоны, а примесный кислород формирует систему уровней вблизи зоны проводимости.

\section{Заключение}

В настоящей работе были изучены спектры ФЛ и спектрально-разрешенные кривые ТЛ в субмикронных кристаллах AlN в виде гексагональных призм. Обнаружено, что зависимости представляют собой широкую полосу с максимумом в области $2.98 \mathrm{eV}$ и могут быть представлены в виде суперпозиции двух полос при 2.43 и $3.0 \mathrm{eV}$. Пик $3.04 \mathrm{eV}$ является доминирующим. Сравнительный анализ полученных данных был выполнен с использованием наших предыдущих результатов и результатов независимых исследований. Показано, что вакансии алюминия $V_{\mathrm{Al}}$ и кислород-вакансионные комплексы $V_{\mathrm{Al}}-\mathrm{O}_{\mathrm{N}}$ являются основными центрами свечения в рассматриваемых специально нелегированных микрокристаллах. В качестве центров захвата электронов выступают вакансии азота $V_{\mathrm{N}}$ с энергией активации $E_{A}=0.45 \mathrm{eV}$.

\section{Финансирование работы}

Исследование выполнено в рамках инициативного научного проекта FEUZ-2020-0059 Минобрнауки РФ и гранта Российского фонда фундаментальных исследований (проект № 18-32-00550), а также при поддержке Постановления 211 Правительства РФ, контракт № 02.A03.21.0006.

\section{Конфликт интересов}

Авторы заявляют, что у них нет конфликта интересов.

\section{Список литературы}

[1] Oyama S.T. The Chemistry of Transition Metal Carbides and Nitrides. London: Springer, Blackie Academic \& Professional, London, 1996. P. 28-52.

[2] Giordano C., Antonietti M. // Nano Today. 2011. V. 6. 4. P. 366. doi 10.1016/j.nantod.2011.06.002

[3] Vokhmintsev A.S., Weinstein I.A., Spiridonov D.M. // J. Lumin. 2012. V. 132. P. 2109. doi 10.1016/j.jlumin.2012.03.066

[4] Вохминцев А.С., Вайнштейн И.А., Спиридонов Д.М., Бекетов Д.А., Бекетов А.Р. // Письма в ЖТФ. 2012. Т. 38. № 4. C. 10. doi 10.1134/S1063785012020319; Vokhmintsev A.S., Weinstein I.A., Spiridonov D.M., Beketov D.A., Beketov A.R. // Tech. Phys. Lett. 2012. V. 38. N 2. P. 160. doi 10.1134/S1063785012020319

[5] Vokhmintsev A., Weinstein I., Spiridonov D. // Phys. Status Solidi C. 2013. V. 10. N 3. P. 457. doi 10.1002/pssc.201200519

[6] Mogilatenko A., Knauer A., Zeimer U., Netzel C., Jeschke J., Unger R.-S., Hartmann C., Wollweber J., Dittmar A., Juda U., Weyers M., Bickermann M. // J. Cryst. Growth. 2019. V. 505. P. 69. doi 10.1016/j.jcrysgro.2018.10.021 
[7] Demol G., Paulmier T., Payan D. // J. Appl. Phys. 2019. V. 125. N 2. P. 025110 . doi $10.1063 / 1.5066434$

[8] Taniyasu Y., Kasu M., Makimoto T. // Nature 2006. V. 441. N 7091. P. 325. doi 10.1038/nature04760

[9] Jung H., Xiong C., Fong K.Y., Zhang X., Tang H.X. // Opt. Lett. 2013. V. 38. N 15. P. 2810. doi 10.1364/OL.38.002810

[10] Shen L., Lv W., Wang N., Wu L., Qi D., Ma Y., Lei $W$. // CrystEngComm. 2017. V. 19. N 39. P. 5940. doi 10.1039/C7CE01335C

[11] Zhao S., Nguyen H.P.T., Kibria M.G., Mi Z.// Prog. Quantum Electron. 2015. V. 44. P. 14. doi 10.1016/j.pquantelec.2015.11.001

[12] Genji K., Uchino T. // Appl. Phys. Lett. 2016. V. 109. P. 021113. doi 10.1063/1.4958891

[13] Weinstein I.A., Vokhmintsev A.S., Chaikin D.V., Afonin Yu.D. // Opt. Mater. 2016. V. 61. P. 111. doi 10.1016/j.optmat.2016.05.054

[14] Shen L., Zhang X., Song J., Li F., Qi D. // J. Mater. Sci.Mater. Electron. 2016. V. 27. P. 12017. doi 10.1007/s10854016-5349-9

[15] Lu H., Schaff W.J., Hwang J., Wu H., Koley G., Eastman L.F. // Appl. Phys. Lett. 2001. V. 79. N 10. P. 1489.

[16] Gregory O.J., Slot A.B., Amons P.S., Crisman E.E. // Surf. Coat. Technol. 1997. V.88. N 1-3. P. 79.

[17] Tsubouchi K., Mikoshiba N. // IEEE Trans. Son. Ultrason. 1985. V. 32. N 5. P. 634.

[18] Fujieda S., Mizuta M., Matsumoto Y. // Adv. Mater. Opt. Electron. 1996. V. 6. N 3. P. 127.

[19] Liu J., Zhang X., Zhang Y., He R., Zhu J. // J. Mater. Res. 2001. V. 16. N 11. P. 3133. doi 10.1557/JMR.2001.0432

[20] Zhang Y., Liu J., He R., Zhang Q., Zhang X., Zhu J. // Chem. Mat. 2001. V. 13. N 11. P. 3899. doi $10.1021 / \mathrm{cm} 001422 \mathrm{a}$

[21] Tondare V.N., Balasubramanian C., Shende S.V., Joag D.S., Godbole V.P., Bhoraskar S.V., Bhadbhade M. // Appl. Phys. Lett. 2002. V. 80. N 25. P. 4813. doi 10.1063/1.1482137.

[22] Bickermann M., Epelbaum B.M., Filip O., Heimann P., Nagata S., Winnacker A. // Phys. Status Solidi B. 2009. V. 246. N 6. P. 1181. doi 10.1002/pssb.200880753

[23] Shen L., Wang N., Xiao X. // Mater. Lett. 2013. V. 94. P. 150. doi 10.1016/j.matlet.2012.12.042

[24] Choudhary R.K., Soni A., Mishra P., Mishra D.R., Kulkarni M.S. // J. Lumin. 2014. V. 155. P. 32. doi 10.1016/j.jlumin.2014.06.016

[25] Vokhmintsev A.S., Weinstein I.A., Chaikin D.V., Spiridonov D.M., Afonin Yu.D. // Funct. Mater. 2014. V. 21. N 1. P. 21. doi $10.15407 / \mathrm{fm} 21.01 .021$

[26] Чайкин Д.В., Афонин Ю.Д., Вайнштейн И.А., Вохминцев А.C., Шульгин Д.В. Заявка на изобретение РФ № 2019112096, 2018; Chaikin D.V., Afonin Yu.D., Weinstein I.A., Vokhmintsev A.S., Shulgin D.B. Russian Federation Patent application № 2019112096, 2018.

[27] Spiridonov D.M., Weinstein I.A., Chaikin D.V., Vokhmintsev A.S., Afonin Yu.D., Chukin A.V. // Radiat. Meas. 2019. V. 122. P. 91. doi 10.1016/j.radmeas.2019.02.001

[28] Vokhmintsev A.S., Minin M.G., Chaykin D.V., Weinstein I.A. // Instrum. Exp. Tech. 2014. V. 57. N 3. P. 369. doi 10.1134/S0020441214020328

[29] Vokhmintsev A.S., Minin M.G., Henaish A.M.A., Weinstein I.A. // Measurement. 2015. V. 66. P. 90. doi 10.1016/j.measurement.2015.01.012
[30] Chaikin D.V., Spiridonov D.M., Vokhmintsev A.S., Martemyanov N.A., Weinstein I.A. // AIP Conf. Proc. 2019. V. 2174. P. 020091 . doi $10.1063 / 1.5134242$

[31] Гурвич А.М. Введение в физическую химию кристаллофосфоров. Учеб. пособие для втузов. М.: Высшая школа, 1971. $336 \mathrm{c}$.

[32] Pasrtňák J., Pačesová S., Roskovcová L. // Czech. J. Phys. 1974. V. 24. P. 1149.

[33] Thapa S.B., Hertkorn J., Scholz F., Prinz G.M., Leute R.A.R., Feneber M., Thonke K., Sauer R., Klein O., Biskupek J., Kaiser U. // J. Cryst. Growth. 2008. V. 310. P. 4939. doi 10.1016/j.jcrysgro.2008.07.091

[34] Nam K.B., Nakarmi M.L., Lin J.Y., Jiang H.X. // Appl. Phys. Lett. 2005. V. 86. P. 222108. doi 10.1063/1.1943489

[35] Koyama T., Sugawara M., Hoshi T., Uedono A., Kaeding J.F., Sharma R., Nakamura S., Chichibu S.F. // Appl. Phys. Lett. 2007. V. 90. P. 241914. doi 10.1063/1.2748315

[36] Koppe T., Hofsäss H., Vetter U. // J. Lumin. 2016. V. 178. P. 267. doi 10.1016/j.jlumin.2016.05.055

[37] Cao Y.G., Chen X.L., Lan Y.C., Li J.Y., Xu Y.P., Xu T., Liu Q.L., Liang J.K. // J. Cryst. Growth. 2000. V. 213. P. 198. doi 10.1016/S0022-0248(00)00379-1

[38] Weinstein I.A., Vokhmintsev A.S., Spiridonov D.M. // Diam. Relat. Mat. 2012. V. 25. P. 59. doi 10.1016/j.diamond.2012.02.004

[39] Nappé J.C., Benabdesselam M., Grosseau Ph., Guilhot D. // Nucl. Instrum. Meth. B. 2011. V. 269. P. 100. doi 10.1016/j.nimb.2010.10.025

[40] Chen R., McKeever S.W.S. Theory of Thermoluminescence and Related Phenomena. World Scientific, Singapore City. 1997. $559 \mathrm{p}$. 